\title{
Numerical Solution of Advection-Diffusion Equation Using Operator Splitting Method
}

\author{
Ersin Bahar $^{a^{*}}$, Gurhan Gurarslan ${ }^{b}$ \\ ${ }^{a, b}$ Pamukkale University, Department of Civil Engineering, Denizli TR-20070, Turkey \\ *E-mail address: ebahar@pau.edu.tr \\ ORCID numbers of authors: \\ 0000-0001-8892-8441 ${ }^{a}$, 0000-0002-9796-3334
}

Received date: November 2017

Accepted date: December 2017

\begin{abstract}
In this study, effects of operator splitting methods to the solution of advection-diffusion equation are examined. Within the context of this work two operator splitting methods, Lie-Trotter and Strang splitting methods were used and comparisons were made through various Courant numbers. These methods have been implemented to advection-diffusion equation in one-dimension. Numerical solutions of advection and dispersion processes were carried out by a characteristics method with cubic spline interpolation (MOC-CS) and Crank-Nicolson (CN) finite difference scheme, respectively. Obtained results were compared with analytical solutions of the problems and available methods in the literature. It is seen that MOC-CS-CN method has lower error norm values than the other methods. MOC-CS-CN produces accurate results even while the time steps are great.
\end{abstract}

Keywords: Advection-diffusion equation, Operator splitting methods, Method of characteristics, Finite difference

\section{Introduction}

Rivers, lakes and other natural waters have been the drain place of urban and industrial wastes since the early days of civilization. In the early days, the amount and composition of these wastes were not at very important levels, so they did not have a negative effect on aquatic environments. However, rapid population growth, rising living standards and the development of the industry have led to an increase in the amount of pollution discharged into the aquatic environment. In order to reduce or eliminate this pollution, the pollutant transport processes represented by the advection-diffusion equation should be well understood and the processes to be carried out should be adapted to the nature of these processes [1]. The one-dimensional mathematical expression of the advection-diffusion equation without the source term is as follows

$$
\frac{\partial C}{\partial t}+U \frac{\partial C}{\partial x}=D_{x} \frac{\partial^{2} C}{\partial x^{2}}
$$

Where $t$ is time, $x$ is spatial coordinate, $C$ is concentration of substance, $U$ is velocity of the flow and $D$ is diffusion coefficient. We denote the spatial and temporal step sizes by $\Delta x$ and $\Delta t$, respectively. Also Courant number, $\mathrm{Cr}$, is computed as $U \Delta t / \Delta x$ and the Peclet number, $\mathrm{Pe}$, is obtained as $U \Delta x / D_{x}$. 
Although advection and diffusion are simultaneous processes, they have very different effects on mass transport. The advection process is only influenced by the past, and it occurs along the characteristic line. However, the diffusion process takes place between the characteristic lines, which will be influenced by both the past and present conditions. This means that there is a need for a numerical method that simultaneously solves both hyperbolic term (advection) and parabolic term (dispersion). There is no numerical method that can completely overcome of this problem [2]. So the great effort has been made on developing the efficient and stable numerical techniques.

Holly and Usseglio-Polatera [3] developed a sensitive numerical method to model the contaminant dispersion in two-dimensional tidal currents. This method uses the approach of high-order bi-cubic Hermite interpolation with characteristics in the solution of the advection part. The Crank-Nicolson scheme was used for the diffusion part in the study. Chen and Falconer [4] used a modified QUICK finite difference scheme for water quality modeling in rivers and coastal waters. Also, they did stability analysis of the modified method. Szymkiewicz [5] solved advection-diffusion equation with the help of Lie-Trotter operator splitting method. Cubic spline interpolation and standard Galerkin finite element method were used for advection and diffusion processes, respectively. Ahmad and Kothyari [6] proposed a new numerical scheme for the solution of the pure advection process. The basis of the proposed method is based on the backward time-line characteristics approach. Tsai et al. [7] investigated effects of the endpoint constraints which are used in the characteristics method with cubic spline interpolation, on the solution of advection process. Verma et al. [8], with the help of Lie-Trotter operator splitting method, used the MacCormack scheme and the Crank-Nicolson finite difference scheme for the solution of the advection and diffusion processes, respectively. Tian and $\mathrm{Ge}$ [9] have developed an exponential fourth-order compact alternating direction implicit method in which Crank-Nicolson scheme used for time discretization and an exponential fourth-order compact difference formula used for spatial discretization. Sari et al. [10] proposed high-order finite difference schemes for the solution of a one-dimensional advection-diffusion equation. Schemes are derived from Taylor series expansion. To get the solutions, they have integrated the fourth-order Runge-Kutta scheme in time with the finite difference schemes up to the tenth order in space. Gurarslan et al. [11] have produced numerical solutions to a onedimensional advection-diffusion equation using a Runge-Kutta scheme of fourth-order and a compact finite difference scheme of sixth-order in space. In the study by Gurarslan [12], numerical simulations of the advection-dispersion equation were performed with high-order compact finite difference schemes. Compact finite difference schemes were used in conjunction with MacCormack and Runge-Kutta schemes to obtain solutions with the accuracy of sixthorder.

In this study, appropriate schemes will be used for the physical structures of the advectiondiffusion problem. Advection process and diffusion process will be solved by characteristics method with cubic spline interpolation (MOC-CS) and the Crank-Nicolson scheme $(\mathrm{CN})$, respectively. These two different methods will be combined through operator splitting methods. For this purpose, first-order Lie-Trotter and second-order Strang-Marchuk operator splitting methods which are frequently used in the literature were chosen and effects of them on the solution will be examined for a one-dimensional problem with sharp structure. The obtained results will be compared with the analytical solution of the test problem and the results in the literature. 


\section{Solution Procedures with Different Operator Splitting Methods}

The mathematical representations of Lie-Trotter and Strang-Marchuk operator splitting methods and when they are applied to advection-diffusion equation, solution procedures will be explained in the next sections.

\subsection{Lie-Trotter Operator Splitting Method}

The Lie-Trotter operator splitting method is a first-order operator splitting method and named as a sequential splitting method. By applying this method to the advection-diffusion equation, the problem is divided into two sub-problems: advection and diffusion. The application of the Lie-Trotter separation method to Eq. (1) is as follows

$$
\begin{aligned}
& \frac{\partial \hat{C}_{1}}{\partial t}+U \frac{\partial \hat{C}_{1}}{\partial x}=0, \quad \hat{C}_{1}\left(t_{n}, x\right)=C\left(t_{n}, x\right), \quad t \in\left[t_{n}, t_{n+1}\right] \\
& \frac{\partial \hat{C}_{2}}{\partial t}=D_{x} \frac{\partial^{2} \hat{C}_{2}}{\partial x^{2}}, \quad \hat{C}_{2}\left(t_{n}, x\right)=\hat{C}_{1}\left(t_{n+1}, x\right), \quad t \in\left[t_{n}, t_{n+1}\right]
\end{aligned}
$$

Where $\hat{C}_{1}$ is the concentration in the advection process and $\hat{C}_{2}$ is the concentration in the diffusion process. Eq. (2) and Eq. (3) represents the pure advection equation and diffusion equation, respectively. In the solution process, Eq. (2) will be solved for a time interval of $\Delta t$ using the initial condition of Eq. (1). The result obtained from there will be the initial condition of Eq. (3). Then Eq. (3) will be solved for a time interval of $\Delta t$ and the solution of Eq. (1) will be obtained for a time interval $\Delta t$. Thus, the problems will be solved consecutively by combining them with the initial conditions [13].

\subsection{Strang-Marchuk Operator Splitting Method}

The Strang-Marchuk operator splitting method is a second-order operator splitting method. By applying this method to Eq. (1), the problem will be divided into two sub-problems, namely advection and diffusion, similar to the Lie-Trotter operator splitting method. But this time these sub-problems will be solved in three steps in total. The application of the Strang-Marchuk opeator splitting method to Eq. (1) is as follows

$$
\begin{gathered}
\frac{\partial \hat{C}_{1}}{\partial t}+U \frac{\partial \hat{C}_{1}}{\partial x}=0, \quad \hat{C}_{1}\left(t_{n}, x\right)=C\left(t_{n}, x\right), \quad t \in\left[t_{n}, t_{n+1 / 2}\right] \\
\frac{\partial \hat{C}_{2}}{\partial t}=D_{x} \frac{\partial^{2} \hat{C}_{2}}{\partial x^{2}}, \quad \hat{C}_{2}\left(t_{n}, x\right)=\hat{C}_{1}\left(t_{n+1 / 2}, x\right), \quad t \in\left[t_{n}, t_{n+1}\right] \\
\frac{\partial \hat{C}_{1}}{\partial t}+U \frac{\partial \hat{C}_{1}}{\partial x}=0, \quad \hat{C}_{1}\left(t_{n+1 / 2}, x\right)=\hat{C}_{2}\left(t_{n+1}, x\right), \quad t \in\left[t_{n+1 / 2}, t_{n+1}\right]
\end{gathered}
$$

Where Eq. (4) will be solved for a time interval of $\Delta t / 2$ using the initial condition of Eq. (1). The solution of Eq. (4) will be used as the initial condition of Eq. (5) and Eq. (5) will be solved for a time interval of $\Delta t$. The obtained result of Eq. (5) will be the initial condition of Eq. (6). Lastly, Eq. (6) will solved for a time interval of $\Delta t / 2$. Thus, the solution of Eq. (1) will be obtained for a time interval of $\Delta t[13]$. 


\section{Method of Characteristics for Advection Process}

The method of characteristics approach is frequently used in the solution of advection process. This is because as the other schemes it does not have time and spatial discretization errors but only the error of the interpolation method. This is achieved by converting the pure advection equation in Eq. (2) into two ordinary differential equations. If both sides of Eq. (2) are multiplied by $d t$, this equation can be written in total derivative form. In this case, Eq. (2) can be represented by the following two ordinary differential equations.

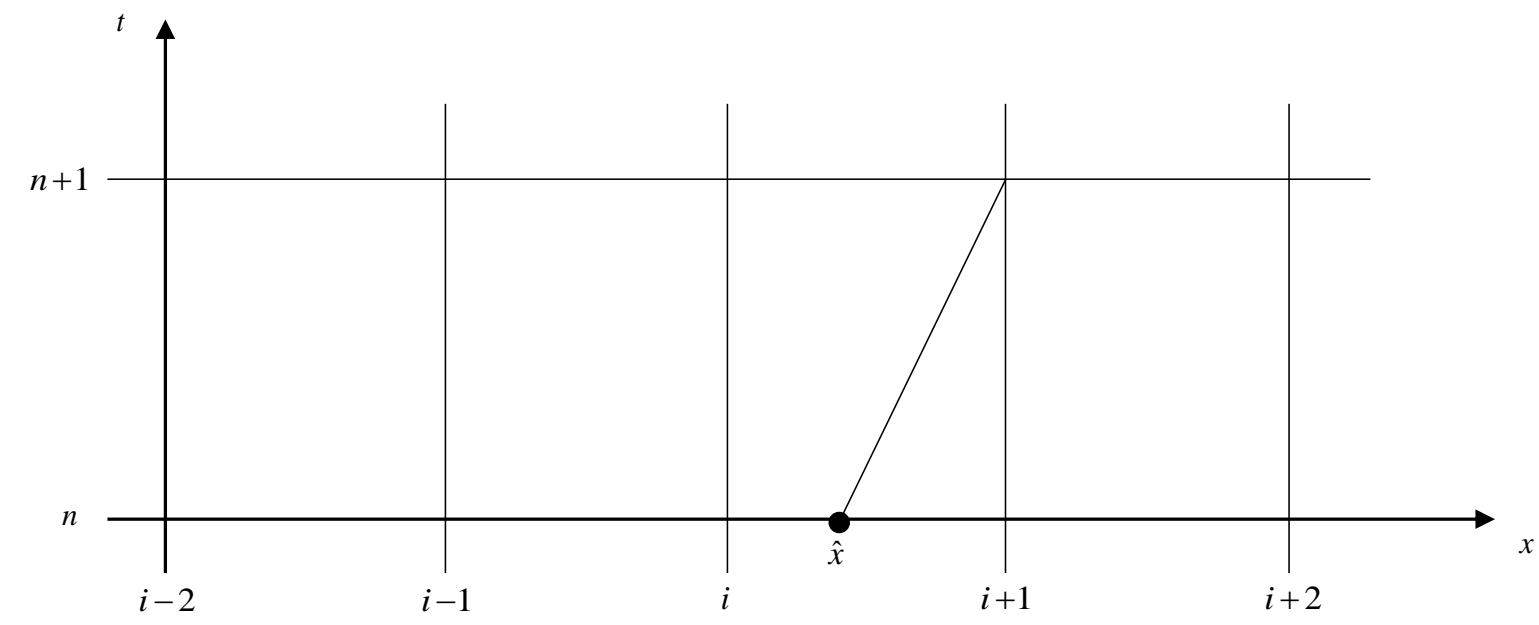

Fig. 1. Finite difference grid structure and trajectory of concentration particle in one-dimension

$$
\begin{aligned}
& \frac{d \hat{C}_{1}}{d t}=0 \\
& \frac{d x}{d t}=U
\end{aligned}
$$

Integration of the Eq. (7) and Eq. (8) yields

$$
\left.\hat{C}_{1}\right|_{\hat{x}, n}=\left.\hat{C}_{1}\right|_{i+1, n+1}
$$

Along

$$
x_{i+1}-\hat{x}=U \Delta t=\operatorname{Cr} \Delta x
$$

When the finite difference grid structure is generated for solution domain as can be seen from Fig. 1, each node representing concentration value can be taken as a concentration particle. We know the concentration particle moves with the velocity of the flow in the advection process, so we can follow its trajectory which is given by Eq. (8) and can be seen in Fig. 1. $\left.\hat{C}_{1}\right|_{i+1, n+1}$ is the concentration value that needs to be calculated. $\left.\hat{C}_{1}\right|_{\hat{x}, n}$ is the concentration value at point $\hat{x}$ between nodes. As we know from Eq. (9) those values are equal. Therefore we need an interpolation method to calculate the concentration value at $\hat{x}$ by using the concentration values at all nodal points at time level $n$. The accuracy of the solution depends on the order of the 
interpolation method. In this study cubic spline interpolation method is picked because of its high-order accuracy [22].

Cubic splines use third-order polynomials generated at each interval for interpolation. Suppose there are $M+1$ data points $\left(x_{1},\left.\hat{C}_{1}\right|_{1, n}\right), \ldots,\left(x_{M+1},\left.\hat{C}_{1}\right|_{M+1, n}\right)$ so that there are $M$ intervals and thus $M$ cubic polynomials. The general expression a cubic polynomial is as follows

$$
P_{i}(x)=\alpha_{i}+\beta_{i}\left(x-x_{i}\right)+\gamma_{i}\left(x-x_{i}\right)^{2}+\theta_{i}\left(x-x_{i}\right)^{3}, \quad i=1,2, \ldots, M
$$

Where $\alpha_{i}, \beta_{i}, \gamma_{i}, \theta_{i}$ are the coefficients that should be calculated. Since we have $M$ cubic polynomials and there are 4 unknown coefficients in each polynomial, we need $4 M$ equations so that these coefficients can be calculated. These equations derived based on the adjacent splines agree at interior knots and also first and second derivatives of the adjacent splines agree at interior knots.

$$
\begin{gathered}
P_{M}\left(x_{M+1}\right)=\left.\hat{C}_{1}\right|_{M+1, n} \\
P_{i}\left(x_{i}\right)=\left.\hat{C}_{1}\right|_{i, n^{\prime}} \quad i=1,2, \ldots, M
\end{gathered}
$$

The established polynomials must provide the concentration values at those points as stated in Eq. (12) and Eq. (13).

$$
\begin{array}{ll}
P_{i}\left(x_{i+1}\right)=P_{i+1}\left(x_{i+1}\right), & i=1,2, \ldots, M-1 \\
P_{i}^{\prime}\left(x_{i+1}\right)=P_{i+1}^{\prime}\left(x_{i+1}\right), & i=1,2, \ldots, M-1 \\
P_{i}^{\prime \prime}\left(x_{i+1}\right)=P_{i+1}^{\prime \prime}\left(x_{i+1}\right), & i=1,2, \ldots, M-1
\end{array}
$$

Eqs. (14-16) represent the equality of the concentration values and first and second derivatives of polynomials at interior knots of adjacent splines. In this way $4 M-2$ equations are created. The last 2 equations that we need will be obtained from the boundary condition. As we know there are various boundary conditions, the natural boundary condition will be used in this study.

$$
\begin{gathered}
P_{1}^{\prime \prime}\left(x_{1}\right)=0 \\
P_{M}^{\prime \prime}\left(x_{M+1}\right)=0
\end{gathered}
$$

Eq. (17) represents the assumptions made in the natural boundary condition. The second derivative in the first and last points equals to zero. Thus $4 \mathrm{M}$ equations are obtained. First, $P_{i}\left(x_{i}\right)=\alpha_{i}(i=1,2, \ldots, M)$ found from Eq. (11). By integrating this with Eq. (13)

$$
\alpha_{i}=\left.\hat{C}_{1}\right|_{i, n^{\prime}} \quad i=1,2, \ldots, M
$$

Eq. (18) obtained. We define the distance between the nodes as $h_{i}=x_{i+1}-x_{i}(i=1,2, \ldots, M)$ and the following equations are obtained when all unknown coefficients are written in $\gamma$. 


$$
\begin{gathered}
\gamma_{1}=0 \\
\gamma_{i-1} h_{i-1}+2 \gamma_{i}\left(h_{i}+h_{i-1}\right)+\gamma_{i+1} h_{i}=K_{1}-K_{2}, \quad i=2,3, \ldots, M \\
\gamma_{M+1}=0
\end{gathered}
$$

Where

$$
\begin{aligned}
& K_{1}=\frac{3\left(\left.\hat{C}_{1}\right|_{i+1, n}-\left.\hat{C}_{1}\right|_{i, n}\right)}{h_{i}} \\
& K_{2}=\frac{3\left(\left.\hat{C}_{1}\right|_{i, n}-\left.\hat{C}_{1}\right|_{i-1, n}\right)}{h_{i-1}}
\end{aligned}
$$

As seen in Eq. (19), the first and last $\gamma$ coefficients are calculated by natural boundary condition. The $\gamma$ coefficients at the other points form a tri-diagonal matrix system consisting of $M-1$ equations. As this system can effectively be solved by the Thomas algorithm, the $\gamma$ coefficients at all points are calculated easily. The values of $\alpha$ coefficients are given in Eq. (18). The calculations of the remaining $\beta$ and $\theta$ coefficients with the help of the $\gamma$ coefficients are as follows

$$
\begin{gathered}
\beta_{i}=\frac{\left.\hat{C}_{1}\right|_{i+1, n}-\left.\hat{C}_{1}\right|_{i, n}}{h_{i}}-\frac{1}{3}\left(2 \gamma_{i}+\gamma_{i+1}\right) h_{i}, \quad i=1,2, \ldots, M \\
\theta_{i}=\frac{\gamma_{i+1}-\gamma_{i}}{3 h_{i}}, \quad i=1,2, \ldots, M
\end{gathered}
$$

Thus, a total of $M$ cubic polynomials are obtained. A detailed description of the arrangements is available from the work of Esfandiari [14].

\section{Crank-Nicolson Scheme for Diffusion Process}

The Crank-Nicolson scheme is an implicit scheme and gives quite accurate results in the solution of the diffusion equation. The application to the diffusion equation given in Eq. (3) is as follows

$$
\begin{gathered}
\frac{\left.\hat{C}_{2}\right|_{i, n+1}-\left.\hat{C}_{2}\right|_{i, n}}{\Delta t} \\
=\frac{D}{2(\Delta x)^{2}}\left(\left.\hat{C}_{2}\right|_{i+1, n}-\left.2 \hat{C}_{2}\right|_{i, n}+\left.\hat{C}_{2}\right|_{i-1, n}\right) \\
+\frac{D}{2(\Delta x)^{2}}\left(\left.\hat{C}_{2}\right|_{i+1, n+1}-\left.2 \hat{C}_{2}\right|_{i, n+1}+\left.\hat{C}_{2}\right|_{i-1, n+1}\right)
\end{gathered}
$$


The values at time level $n$ are known and the values at time level $n+1$ are unknown. By gathering the known values to the right side and the unknown values to the left side in the Eq. (24), the following equation is obtained.

$$
\left.a_{i} \hat{C}_{2}\right|_{i-1, n+1}+\left.b_{i} \hat{C}_{2}\right|_{i, n+1}+\left.c_{i} \hat{C}_{2}\right|_{i+1, n+1}=f_{i}
$$

The coefficients in Eq. (25)

$$
\begin{gathered}
a_{i}=\frac{D \Delta t}{2(\Delta x)^{2}} \\
b_{i}=-\frac{D \Delta t}{(\Delta x)^{2}}-1 \\
c_{i}=\frac{D \Delta t}{2(\Delta x)^{2}} \\
f_{i}=-\left.\hat{C}_{2}\right|_{i, n}-\frac{D \Delta t}{2(\Delta x)^{2}}\left(\left.\hat{C}_{2}\right|_{i+1, n}-\left.2 \hat{C}_{2}\right|_{i, n}+\left.\hat{C}_{2}\right|_{i-1, n}\right)
\end{gathered}
$$

Eq. (25) forms a tridiagonal system of equations. This system can be solved effectively by the Thomas algorithm [15].

\section{Numerical Application}

In this section, a sharp structure one-dimensional advection-diffusion equation will be solved with different operator splitting methods which combine MOC-CS and CN. Effects of operator splitting methods will be examined for various Courant numbers. Obtained results will be compared with solutions available in the literature and exact solution. In addition, the accuracy of the methods will be evaluated by calculating the error norms. The error norms are calculated as follows.

$$
\begin{aligned}
& L_{\infty}=\max _{i}\left|C_{i}^{\text {exact }}-C_{i}^{\text {numerical }}\right| \\
& L_{2}=\sqrt{\sum_{i=1}^{M+1}\left|C_{i}^{\text {exact }}-C_{i}^{\text {numerical }}\right|^{2}}
\end{aligned}
$$

Example: Velocity of the flow and diffusion coefficient are taken as $U=0.01 \mathrm{~m} / \mathrm{s}$ and $D=$ $0.002 \mathrm{~m}^{2} / \mathrm{s}$ in this experiment. Length of the channel picked as $L=100 \mathrm{~m}$. The analytic solution of this problem can be obtained by solving the following equation [5].

$$
C(x, t)=\frac{1}{2} \operatorname{erfc}\left(\frac{x-U t}{\sqrt{4 D t}}\right)+\frac{1}{2} \exp \left(\frac{U x}{D}\right) \operatorname{erfc}\left(\frac{x+U t}{\sqrt{4 D t}}\right)
$$


The used boundary conditions in the solution are as follows

$$
\begin{gathered}
C(0, t)=1 \\
-D\left(\frac{\partial C}{\partial x}\right)(L, t)=0
\end{gathered}
$$

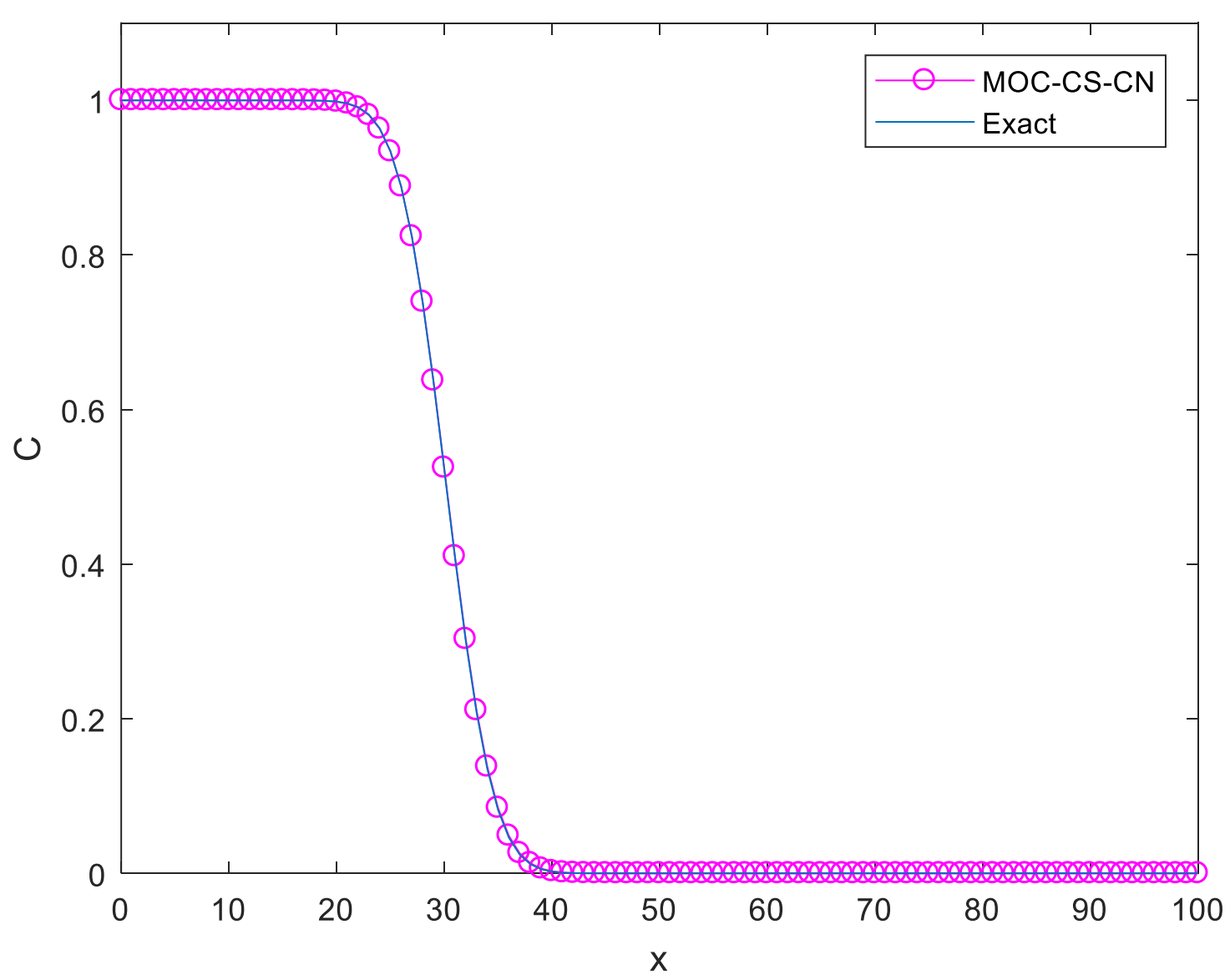

Fig. 2. Comparison of the exact and the numerical solution obtained with MOC-CS-CN method for $\Delta x=1 \mathrm{~m}$ and $\Delta t=10 \mathrm{~s}$

Initial condition of the problem can be obtained from the exact solution. In all calculations spatial step size picked as $\Delta x=1 \mathrm{~m}$. Fig. 2 shows that comparison of exact solution and numerical solution with MOC-CS-CN method for time interval of $\Delta t=10$. As it clearly can be seen there is an excellent agreement between numerical solution and exact solution. As shown in Fig. 2, when the maximum calculation time is $3000 \mathrm{~s}$, the critical concentration values are between 18 and $42 \mathrm{~m}$. Therefore, the values at these points will be compared.

Since the problem has a sharp structure, obtaining close results to the analytical solution is very difficult. For this reason, calculations start from a small time interval. The calculations in Table 1 were made for $\Delta t=1$. Almost all of the methods have found the same values as the analytical solution. There is also no visible difference between operator splitting methods. For this reason, the time interval will be gradually enlarged until this difference appears to be clear.

The calculations in Table 2 were made for $\Delta t=10$. This small change in time interval is enough for other methods to start to get away from the analytical solution. When the calculated concentration values are compared it seems clear that the MOC-CS-CN method has the closest 
results to the analytical solution and lowest error norms for both operator splitting methods. Thus, it has become clear how effective is the MOC-CS-CN method in sharp problems. In addition, the effect of operator splitting methods on the solution seems to appear.

Table 1. Comparison of obtained solution with exact and numerical solutions in the literature

\begin{tabular}{|c|c|c|c|c|c|c|}
\hline \multirow[b]{2}{*}{$\mathrm{x}(\mathrm{m})$} & \multirow{2}{*}{$\begin{array}{l}{[12]} \\
\text { MC- } \\
\text { CD6 }\end{array}$} & \multirow{2}{*}{$\begin{array}{c}{[11]} \\
\text { RK4-CD6 }\end{array}$} & \multirow{2}{*}{$\begin{array}{c}{[16]} \\
\text { CuTBSM }\end{array}$} & \multicolumn{2}{|c|}{ MOC-CS-CN } & \multirow[b]{2}{*}{ Exact } \\
\hline & & & & Lie-Trotter & Strang & \\
\hline 0 & 1.000 & 1.000 & 1.000 & 1.000 & 1.000 & 1.000 \\
\hline 18 & 1.000 & 1.000 & 1.000 & 1.000 & 1.000 & 1.000 \\
\hline 19 & 0.999 & 0.999 & 0.999 & 0.999 & 0.999 & 0.999 \\
\hline 20 & 0.998 & 0.998 & 0.998 & 0.998 & 0.998 & 0.998 \\
\hline 21 & 0.996 & 0.996 & 0.996 & 0.996 & 0.996 & 0.996 \\
\hline 22 & 0.991 & 0.991 & 0.991 & 0.990 & 0.990 & 0.991 \\
\hline 23 & 0.982 & 0.982 & 0.982 & 0.981 & 0.981 & 0.982 \\
\hline 24 & 0.964 & 0.964 & 0.964 & 0.963 & 0.963 & 0.964 \\
\hline 25 & 0.935 & 0.934 & 0.934 & 0.933 & 0.933 & 0.934 \\
\hline 26 & 0.889 & 0.889 & 0.888 & 0.888 & 0.888 & 0.889 \\
\hline 27 & 0.824 & 0.823 & 0.822 & 0.823 & 0.823 & 0.823 \\
\hline 28 & 0.739 & 0.738 & 0.736 & 0.738 & 0.738 & 0.738 \\
\hline 29 & 0.637 & 0.636 & 0.635 & 0.635 & 0.635 & 0.636 \\
\hline 30 & 0.523 & 0.523 & 0.522 & 0.522 & 0.522 & 0.523 \\
\hline 31 & 0.408 & 0.408 & 0.408 & 0.408 & 0.408 & 0.408 \\
\hline 32 & 0.301 & 0.301 & 0.301 & 0.301 & 0.301 & 0.301 \\
\hline 33 & 0.208 & 0.208 & 0.208 & 0.209 & 0.209 & 0.208 \\
\hline 34 & 0.135 & 0.135 & 0.136 & 0.137 & 0.137 & 0.135 \\
\hline 35 & 0.082 & 0.082 & 0.082 & 0.084 & 0.084 & 0.082 \\
\hline 36 & 0.047 & 0.046 & 0.046 & 0.048 & 0.048 & 0.046 \\
\hline 37 & 0.025 & 0.024 & 0.024 & 0.026 & 0.026 & 0.024 \\
\hline 38 & 0.012 & 0.012 & 0.012 & 0.013 & 0.013 & 0.012 \\
\hline 39 & 0.005 & 0.005 & 0.005 & 0.006 & 0.006 & 0.005 \\
\hline 40 & 0.002 & 0.002 & 0.002 & 0.003 & 0.003 & 0.002 \\
\hline 41 & 0.001 & 0.001 & 0.001 & 0.001 & 0.001 & 0.001 \\
\hline 42 & 0.000 & 0.000 & 0.000 & 0.000 & 0.000 & 0.000 \\
\hline $\mathrm{L}_{2}$ & 0.0017 & 0.0017 & - & 0.0046 & 0.0046 & - \\
\hline $\mathrm{L}_{\infty}$ & 0.0008 & 0.0008 & - & 0.0019 & 0.0019 & - \\
\hline
\end{tabular}

The calculated $L_{\infty}$ error values are compared with other errors in the literature for different time intervals in Table 3. Except for the error of extended cubic B-spline collocation method at $\Delta t=$ $1 \mathrm{~s}$, MOC-CS-CN always has smaller error values. In addition, the increase of the time interval makes the effect of the operator splitting method more noticeable. It is clear that the Strang splitting method improves the quality of the solution. 
Table 2. Comparison of obtained solution with exact and numerical solutions in the literature

\begin{tabular}{|c|c|c|c|c|c|c|}
\hline & & & & & & \\
\hline & {$[12]$} & [11] & [16] & MOC-CS & $-\mathrm{CN}$ & \\
\hline x (m) & $\begin{array}{l}\text { MC- } \\
\text { CD6 }\end{array}$ & RK4-CD6 & CuTBSM & Lie-Trotter & Strang & Exact \\
\hline 0 & 1.000 & 1.000 & 1.000 & 1.000 & 1.000 & 1.000 \\
\hline 18 & 1.000 & 1.000 & 1.000 & 1.000 & 1.000 & 1.000 \\
\hline 19 & 0.999 & 0.999 & 0.999 & 0.999 & 0.999 & 0.999 \\
\hline 20 & 0.998 & 0.998 & 0.998 & 0.998 & 0.998 & 0.998 \\
\hline 21 & 0.996 & 0.996 & 0.996 & 0.996 & 0.996 & 0.996 \\
\hline 22 & 0.991 & 0.992 & 0.991 & 0.991 & 0.991 & 0.991 \\
\hline 23 & 0.982 & 0.982 & 0.982 & 0.981 & 0.981 & 0.982 \\
\hline 24 & 0.965 & 0.965 & 0.963 & 0.963 & 0.963 & 0.964 \\
\hline 25 & 0.936 & 0.936 & 0.933 & 0.934 & 0.934 & 0.934 \\
\hline 26 & 0.891 & 0.891 & 0.885 & 0.889 & 0.889 & 0.889 \\
\hline 27 & 0.827 & 0.827 & 0.818 & 0.824 & 0.824 & 0.823 \\
\hline 28 & 0.743 & 0.743 & 0.732 & 0.739 & 0.739 & 0.738 \\
\hline 29 & 0.642 & 0.641 & 0.631 & 0.637 & 0.637 & 0.636 \\
\hline 30 & 0.529 & 0.528 & 0.517 & 0.525 & 0.524 & 0.523 \\
\hline 31 & 0.414 & 0.413 & 0.404 & 0.410 & 0.410 & 0.408 \\
\hline 32 & 0.306 & 0.306 & 0.298 & 0.303 & 0.302 & 0.301 \\
\hline 33 & 0.213 & 0.212 & 0.207 & 0.211 & 0.210 & 0.208 \\
\hline 34 & 0.138 & 0.138 & 0.134 & 0.138 & 0.138 & 0.135 \\
\hline 35 & 0.084 & 0.084 & 0.081 & 0.085 & 0.084 & 0.082 \\
\hline 36 & 0.048 & 0.048 & 0.045 & 0.049 & 0.049 & 0.046 \\
\hline 37 & 0.025 & 0.025 & 0.023 & 0.026 & 0.026 & 0.024 \\
\hline 38 & 0.012 & 0.012 & 0.011 & 0.013 & 0.013 & 0.012 \\
\hline 39 & 0.006 & 0.006 & 0.005 & 0.006 & 0.006 & 0.005 \\
\hline 40 & 0.002 & 0.002 & 0.002 & 0.003 & 0.003 & 0.002 \\
\hline 41 & 0.001 & 0.001 & 0.001 & 0.001 & 0.001 & 0.001 \\
\hline 42 & 0.000 & 0.000 & 0.000 & 0.000 & 0.000 & 0.000 \\
\hline $\mathrm{L}_{2}$ & 0.0148 & 0.0142 & - & 0.0073 & 0.0064 & - \\
\hline $\mathrm{L}_{\infty}$ & 0.0060 & 0.0055 & - & 0.0028 & 0.0025 & - \\
\hline
\end{tabular}

Table 3. Comparison of $L_{\infty}$ error norms $(\Delta x=1 \mathrm{~m})$

\begin{tabular}{ccccc}
\hline & \multicolumn{2}{c}{$[17]$} & \multicolumn{2}{c}{ MOC-CS-CN } \\
$\Delta \mathrm{t}(\mathrm{s})$ & BSCM & ECuBSCM & $\begin{array}{c}\text { Lie- } \\
\text { Trotter }\end{array}$ & Strang \\
\hline 60 & 0.04330 & 0.04250 & 0.01942 & 0.01180 \\
30 & 0.01962 & 0.01961 & 0.00828 & 0.00567 \\
20 & 0.01270 & 0.01260 & 0.00512 & 0.00376 \\
10 & 0.00685 & 0.00608 & 0.00284 & 0.00251 \\
5 & 0.00409 & 0.00307 & 0.00222 & 0.00212 \\
1 & 0.00224 & 0.00127 & 0.00188 & 0.00187 \\
\hline
\end{tabular}


The calculations in Table 4 were made for $\Delta t=60$. Examining Table 4 , it can be seen that none of the methods have found close results to the exact solution. This indicates that selected time interval is quite large. When the results of the operator splitting methods are compared, the improvement provided by the Strang operator splitting method is clearly visible. But this improvement was not enough.

Table 4. Comparison of obtained solution with exact and numerical solutions in the literature

\begin{tabular}{|c|c|c|c|c|c|c|c|c|c|c|}
\hline \multirow{3}{*}{$\mathrm{x}(\mathrm{m})$} & \multirow{3}{*}{$\begin{array}{c}{[18]} \\
\text { MOCS }\end{array}$} & \multirow{3}{*}{$\begin{array}{c}{[5]} \\
\text { MOCG }\end{array}$} & \multirow{3}{*}{$\begin{array}{c}\text { [19] } \\
\text { CBSG }\end{array}$} & \multicolumn{4}{|c|}{$(\Delta t=60 \mathrm{~s})$} & \multirow{2}{*}{\multicolumn{2}{|c|}{ MOC-CS-CN }} & \multirow{3}{*}{ Exact } \\
\hline & & & & \multicolumn{2}{|c|}{$[20]$} & \multicolumn{2}{|c|}{$[21]$} & & & \\
\hline & & & & FEMLSF & FEMQSF & $\mathrm{TC}$ & TG & \multicolumn{2}{|c|}{ IN-Trotter Strang } & \\
\hline 0 & 1.000 & 1.000 & 1.000 & 1.000 & 1.000 & 1.000 & 1.000 & 1.000 & & 1.000 \\
\hline 18 & 1.000 & & & & & 1.000 & & & & 1.000 \\
\hline 19 & 1.000 & 999 & 1.000 & & & 0.999 & 0.999 & 0.999 & 0.999 & 0.999 \\
\hline 20 & 1.000 & 0.998 & 0.999 & & 000 & 0.999 & 0.9 & 0.998 & 8 & 0.998 \\
\hline 21 & 1.000 & & & & & & & & & .996 \\
\hline 22 & 1.000 & .990 & 0.991 & & 996 & 0.998 & 0.9 & 0.992 & 0.991 & 0.991 \\
\hline 23 & 1.000 & 0.978 & 0.981 & & & 994 & & & & 0.982 \\
\hline 24 & 1.000 & & 0.961 & & 0.974 & 0.987 & 0.960 & 0.967 & 0.965 & 0.964 \\
\hline 25 & 1.000 & & 0.9 & & & 972 & & & & 0.934 \\
\hline 26 & 0.996 & 0.870 & $0.8^{\prime}$ & & & 0.945 & 0.8 & 897 & & 0.889 \\
\hline 27 & 13 & & 0.80 & & & 902 & & & & 0.823 \\
\hline 28 & 1.047 & & 0.7 & & & 838 & 0.7 & & & 0.738 \\
\hline 29 & 397 & 602 & 0.59 & & & 0.755 & & 0 & & 0.636 \\
\hline 30 & & & & & & & & & & \\
\hline 31 & 0.067 & 0.375 & 0.366 & & & 0.541 & 0.3 & 0.427 & 0.4 & 0.408 \\
\hline 32 & & & & & & & & 0.318 & & 0.301 \\
\hline 33 & -0.010 & & 0.1 & & & 0.320 & 0.1 & 0.222 & & 0.208 \\
\hline 34 & 0.002 & 0.118 & 0.118 & & 0.147 & 0.227 & 0.1 & 0.146 & 0.143 & 0.135 \\
\hline 35 & 0.000 & & 0.072 & & & 0.152 & 0.072 & & & 0.082 \\
\hline 36 & 0.000 & .038 & 0.042 & & & 0.096 & 0.041 & 0.052 & & 0.046 \\
\hline 37 & 0.000 & & & & & 0.057 & 0.023 & & & 0.024 \\
\hline 38 & 0.000 & 0.009 & 0.012 & & & 0.032 & 0.012 & & & 0.012 \\
\hline 39 & 0.000 & & & & & & & & & 0.005 \\
\hline 40 & 0.000 & 0.002 & 0.003 & 0.003 & 0.005 & 0.008 & 0.002 & 0.003 & 0.003 & 0.002 \\
\hline 41 & 0.000 & 0.001 & 0.001 & & & 0.004 & & & & 0.001 \\
\hline 42 & 0.000 & 0.000 & 0.001 & 0.000 & 0.001 & 0.001 & 0.000 & 0.001 & 0.000 & 0.000 \\
\hline $\mathrm{L}_{2}$ & - & - & - & - & - & - & - & 0.0479 & 0.0300 & - \\
\hline $\mathrm{L}_{\infty}$ & - & - & - & - & - & - & - & 0.0194 & 0.0118 & - \\
\hline
\end{tabular}

\section{Conclusions}

This paper deals with the solution of advection-diffusion equation based on operator splitting approach. Two different operator splitting methods were used such as Lie-Trotter and StrangMarchuk. The solutions of advection process and diffusion process were obtained by the method of characteristics with cubic spline and Crank-Nicolson scheme, respectively. These two different methods integrated through operator splitting methods and effects of them on the solution examined by a one-dimensional advection-diffusion problem which has a sharp 
structure. The examination has been done by comparing error norm values and comparison with other results available in the literature. As a result, the MOC-CS-CN method achieved very good results. It has almost identical results when $\Delta t$ is small. Also it has been observed that the Strang operator splitting method improves the quality of the result when the time interval increases. In future studies, it is considered to extend this method to multi-dimensional problems by operator splitting methods.

\section{References}

[1] Srivastava, R., Flow Through Open Channels, Oxford University Press, 2008.

[2] Baptista, A. E. De M., Solution of Advection-Dominated Transport by EulerianLagrangian Methods Using the Backward Method of Characteristics, Ph.D Thesis, MIT, Cambridge, 1987.

[3] Holly, F.M., Usseglio-Polatera, J., Dispersion simulation in two-dimensional tidal flow. Journal of Hydraulic Engineering, 110(7), 905-926, 1984.

[4] Chen, Y., Falconer, R.A., Advection-diffusion modelling using the modified QUICK scheme. International Journal for Numerical Methods in Fluids, 15(10), 1171-1196, 1992.

[5] Szymkiewicz, R., Solution of the advection-diffusion equation using the spline function and finite elements. Communications in Numerical Methods in Engineering, 9, 197-206, 1993.

[6] Ahmad, Z., Kothyari, U.C., Time-line cubic spline interpolation scheme for solution of advection equation. Computers and Fluids, 30(6), 737-752, 2001.

[7] Tsai, T.L., Yang, J.C., Huang, L.H., Characteristics method using cubic-spline interpolation for advection-diffusion equation. Journal of Hydraulic Engineering, 130(6), 580-585, 2004.

[8] Verma, P., Prasad, K.S.H., Ojha, C.S.P., MacCormack scheme based numerical solution of advection-dispersion equation. ISH Journal of Hydraulic Engineering, 12(1), 27-38, 2006.

[9] Tian, Z.F., Ge, Y.B., A fourth-order compact ADI method for solving two-dimensional unsteady convection-diffusion problems. Journal of Computational and Applied Mathematics, 198(1), 268-286, 2007.

[10] Sari, M., Gürarslan, G., Zeytinoğlu, A., High-order finite difference schemes for solving the advection-diffusion equation. Mathematical and Computational Applications, 15(3), 449-460, 2010.

[11] Gurarslan, G., Karahan, H., Alkaya, D., Sari, M., Yasar, M., Numerical solution of advection-diffusion equation using a sixth-order compact finite difference method. Mathematical Problems in Engineering, vol. 2013, Article ID 672936, 7 pages, 2013.

[12] Gurarslan, G., Accurate simulation of contaminant transport using high-order compact finite difference schemes. Journal of Applied Mathematics, 2014, 1-8, 2014.

[13] Hundsdorfer, W., Verwer, J., Numerical Solution of Time-Dependent Advection-DiffusionReaction Equations, Springer-Verlag Berlin Heidelberg, 2003.

[14]Esfandiari, R.S., Numerical Methods for Engineers and Scientists Using MATLAB, New York: Taylor and Francis Group, 2013.

[15]Lam, C.Y., Applied Numerical Methods for Partial Differential Equations, Singapore, Simon and Schuster, 1994.

[16]Nazir, T., Abbas, M., Izani, A., Abd, A., The numerical solution of advection-diffusion problems using new cubic trigonometric B-splines approach. Applied Mathematical Modelling, 40(7-8), 4586-4611, 2016.

[17] Irk, D., Dag, I., Tombul, M., Extended cubic B-spline solution of the advection-diffusion equation. KSCE Journal of Civil Engineering, 19(4), 929-934, 2015. 
[18] Holly, F.M., Preissmann, A., Accurate calculation of transport in two dimensions. Journal of Hydraulic Division, 103(11), 1259-1277, 1977.

[19] Gardner, L.R.T. and Dag, I., A Numerical Solution of the Advection-Diffusion Equation Using B-Spline Finite Element, Proceedings International AMSE Conference, Lyon, France, 109-116, 1994.

[20]Dag, I., Irk, D., Tombul, M., Least-squares finite element method for the advectiondiffusion equation. Applied Mathematics and Computation, 173(1), 554-565, 2006.

[21]Dag, I., Canivar, A., Sahin, A., Taylor-Galerkin method for advection-diffusion equation. Kybernetes, 40(5/6), 762-777, 2011.

[22] Bahar, E., Numerical Solution of Advection-Dispersion Equation Using Operator Splitting Method, Denizli: Pamukkale University, (in Turkish) 2017. 